\title{
Untersuchungen zur synchronen oder zweistufigen Fragmentierung der Molekül-Ionen von 3-substituierten Tropanen ${ }^{1)}$
}

\author{
Hans-Fr. Grützmacher* und Gerda Lange \\ Fakultät für Chemie der Universität Bielefeld, \\ Universitätsstraße, D-4800 Bielefeld 1
}

Eingegangen am 19. September 1977

\begin{abstract}
Die Fragmentierung der Molekül-Ionen von stereoisomeren Tropanen mit verschiedenen Substituenten in Position $3(1-11)$ zu den Ionen $m / e=124$ durch Abspaltung des Substituenten wird untersucht. Durch Vergleich der MIKE-Spektren und durch $A$ (Ion)-Messungen wird gezeigt, daß unabhängig von der Konfiguration an $C(3)$ die Ionen a entstehen. Es werden keine Hinweise für eine synchrone Spaltung der $\mathrm{C}(1)-\mathrm{C}(2)$ - und der $\mathrm{C}(3)-\mathrm{X}$-Bindung entsprechend einer Fragmentierung nach Grob erhalten, sondern die Ionen a entstehen in einem zweistufigen ProzeB, beginnend mit einer $\alpha$-Spaltung der Molekül-Ionen. Der Zerfall unterliegt keinen stereoelektronischen Bedingungen; die Intensität der Ionen a kann daher nicht zu einer einfachen massenspektrometrischen Identifizierung von $3 \alpha$ - und $3 \beta$-Tropanderivaten benutzt werden.
\end{abstract}

\section{Investigation of a Synchronous or Two Step Fragmention of Molecular Ions of 3-Substituted Tropanes}

The fragmentation of molecular ions of stereoisomeric tropanes with different substituents at position 3 has been studied. It is shown by comparison of MIKE-spectra and $A$ (ion)-measurements, that the ions a are formed independently from the configuration at $\mathrm{C}(3)$. No indications of a synchronous cleavage of the bonds $\mathrm{C}(1)-\mathrm{C}(2)$ and $\mathrm{C}(3)-\mathrm{X}$ are obtained, but the ions a arise from a two step process, starting with $\alpha$-cleavage of the molecular ions. This fragmentation is not governed by stereoelectronic requirements, thus the intensities of ions a cannot be used for a mass spectrometric identification of $3 \alpha$ - and $3 \beta$-tropane derivatives.

Es ist vermutet worden ${ }^{2)}$, daß die Molekül-Ionen organischer Verbindungen im Massenspektrometer nach den Mechanismen der Fragmentierung von $C$. A. Grob zerfallen können. Uns interessierte dieses Problem im Zusammenhang mit Untersuchungen über sterische Effekte in den Massenspektren. Für die Grob'sche Fragmentierung ist bekannt ${ }^{3)}$, daß sie bei anti-paralleler Anordnung der beteiligten Bindungen synchron und um mehrere Größenordnungen schneller erfolgt als bei einer zweistufigen Fragmentierung. Stereoisomere cyclischer und bicyclischer Verbindungen unterscheiden sich daher häufig drastisch durch die Fragmentierungsgeschwindigkeiten bei solvolytischen Reaktionen. Wenn die gleichen stereoelektronischen Bedingungen auch für eine analoge Fragmentierung von Molekül-Ionen in der Gasphase gelten, sollte es möglich sein, Stereo-

1) Mechanismus massenspektrometrischer Fragmentierungsreaktionen, XX. - XIX. Mitteil.:

D. Kuck und H. Fr. Grützmacher, Org. Mass Spectrom. 13, 90 (1978).

2) W. J. Richter und W. Vetter, Org. Mass Spectrom. 2, 781 (1969).

3) C. A. Grob, Angew. Chem. 81, 543 (1969); Angew. Chem., Int. Ed. Engl. 8, 535 (1969).

(C) Verlag Chemie, GmbH, D-6940 Weinheim, 1978 
isomere geeigneter Struktur an Hand von vorhersagbaren Effekten in den Massenspektren zu unterscheiden.

Für diese Untersuchungen wurden die Stereoisomeren von 3-substituierten Tropanen ausgewählt. Die $\beta$-Isomeren dieser Verbindungen besitzen das $a-b-c-d-X-S y s t e m$ der Grob'schen Fragmentierung in einer fixierten antiparallelen Anordnung, während bei den $\alpha$-Isomeren wegen der axialen Stellung des 3-Substituenten diese Anordnung nur in der energiereichen Bootkonformation möglich ist. Beim 3-Chlortropan ist daher die Geschwindigkeit der Fragmentierung unter solvolytischen Bedingungen bei $100^{\circ} \mathrm{C}$ für das $\beta$-Isomere auch um den Faktor von ca. $10^{3}$ größer als für das $\alpha$-Derivat ${ }^{4)}$.

Die 70-eV-Massenspektren des 3-Tropanols und einiger Derivate sind bekannt; die Fragment-Ionen-Bildung ist mit Hilfe deuterierter Derivate untersucht worden ${ }^{5}$. Auffallend an den Massenspektren des 3-Tropanols und seiner Ester ist die Abspaltung des Substituenten in Stellung 3 aus den Molekül-Ionen zu $\mathrm{C}_{8} \mathrm{H}_{14} \mathrm{~N}^{+}$-Ionen $m / e=124$, obwohl die Molekül-Ionen cyclischer Alkohole und ihrer Ester sonst bevorzugt $\mathrm{H}_{2} \mathrm{O}$ bzw. $\mathrm{HO}_{2} \mathrm{C}-\mathrm{R}$ eliminieren. Dieser Effekt kann als Folge einer Grob'schen Fragmentierung gedeutet werden, so daß die Ionen $m / e=124$ die Struktur a besitzen.

Die bei den Molekül-Ionen von Cyclohexanol und seinen Derivaten bevorzugte 1,4Eliminierung von $\mathrm{H}_{2} \mathrm{O}$ bzw. HOR kann jedoch beim 3-Tropanol wegen der 1,4-Stellung von N-Atom und Substituent nicht eintreten. Es ist möglich, daß statt dessen die Spaltung der $\mathrm{C}(3)-\mathrm{X}$-Bindung durch einen transanularen Effekt des $\mathrm{N}$-Atoms unterstützt wird. In diesem Fall sollten Ionen $m / e=124$ mit der Struktur b entstehen ${ }^{5 a}$.

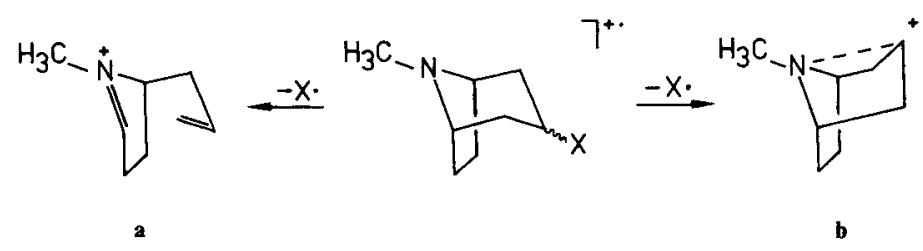

Es wurden die $\alpha, \beta$-Isomerenpaare $\mathbf{1} \mathbf{a}, \mathbf{b}-\mathbf{1 1} \mathbf{a}, \mathbf{b}$ von verschieden 3 -substituiertem Tropan hergestellt und massenspektrometrisch untersucht, um einen eventuellen Einfluß der Abgangsgruppe $\mathrm{X}$ auf die Bildung der Ionen $m / e=124 \mathrm{zu}$ erkennen. Die Konfiguration der Verbindungen an C(3) ist durch den Syntheseweg bekannt und wurde NMR-spektroskopisch an Hand der Signalform und Signalbreite für das Proton an C(3) überprüft.

Für den Nachweis eines Zerfalls der Molekül-Ionen von 1-11 durch eine Grob'sche Fragmentierung sind Informationen über die Struktur der Ionen $m / e=124$ in den Massenspektren der verschiedenen Verbindungen wichtig. Außer durch klassische massenspektroskopische Methoden wie die Untersuchung isotopmarkierter Verbindungen, die im vorliegenden Fall jedoch nicht anwendbar ist, und die Messung von Auftrittspotentialen $A$ (Ion) können derartige Hinweise durch eine Analyse der Zerfallsprodukte von Ionen $m / e=124$ bei Stoßaktivierung (CA-Massenspektren) oder von metastabilen

4) A. T. Bottini, C. A. Grob, E. Schumacher und J. Zergenyi, Helv. Chim. Acta 49, 2516 (1966).

5) 5a) J. Parello, P. Longevialle, W. Vetter und J. A. Mc Closky, Bull. Soc. Chim. Fr. 1963, 2787. -

5b) E. C. Blossey, H. Budzikiewicz, M. Ohashi, G. Fodor und C. Djerassi, Tetrahedron 20, 584 (1964). $-{ }^{5 \mathrm{c})}$ D. H. Smith, A. M. Duffield und C. Djerassi, Org. Mass Spectrom. 7, 367 (1973). 
Ionen $m / e=124$ (MIKE-Spektren, MIKE = Mass analyzed Ion $K$ inetic Energy) erhalten werden. Die CA-Massenspektren ${ }^{6)}$ der untersuchten Ionensorte sind für die Ionen etwa so strukturspezifisch wie normale Massenspektren für Moleküle organischer Verbindungen, da die Fragmentierung der ausgewählten Ionen bei der Stoßanregung mit neutralen Gasmolekülen ähnlichen Regeln folgt wie bei einer Anregung durch Elektronenstoß.

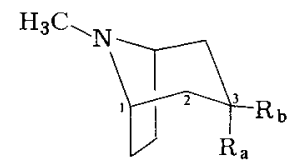

\begin{tabular}{l|lll|ll} 
& $\mathrm{R}_{\mathrm{a}}$ & $\mathrm{R}_{\mathrm{b}}$ & & $\mathrm{R}_{\mathbf{a}}$ & $\mathrm{R}_{\mathbf{b}}$ \\
\hline $\mathbf{1 a}$ & $\mathrm{OH}$ & $\mathrm{H}$ & $\mathbf{7 a}$ & $\mathrm{OSO}_{2} \mathrm{CH}_{3}$ & $\mathrm{H}$ \\
$\mathbf{1 b}$ & $\mathrm{H}$ & $\mathrm{OH}$ & $\mathbf{7 b}$ & $\mathrm{H}$ & $\mathrm{OSO}_{2} \mathrm{CH}_{3}$ \\
$\mathbf{2 a}$ & $\mathrm{Cl}$ & $\mathrm{H}$ & $\mathbf{8 a}$ & $\mathrm{OCONHCH}_{3}$ & $\mathrm{H}$ \\
$\mathbf{2 b}$ & $\mathrm{H}$ & $\mathrm{Cl}$ & $\mathbf{8 b}$ & $\mathrm{H}$ & $\mathrm{OCONHCH}_{3}$ \\
$\mathbf{3 a}$ & $\mathrm{Br}$ & $\mathrm{H}$ & $\mathbf{9 a}$ & $\mathrm{OCONHC}_{6} \mathrm{H}_{5}$ & $\mathrm{H}$ \\
$\mathbf{3 b}$ & $\mathrm{H}$ & $\mathrm{Br}$ & $\mathbf{9 b}$ & $\mathrm{H}$ & $\mathrm{OCONHC}_{6} \mathrm{H}_{5}$ \\
$\mathbf{4 a}$ & $\mathrm{OCH}_{\mathbf{3}}$ & $\mathrm{H}$ & $\mathbf{1 0 a}$ & $\mathrm{OCSNHC}_{6} \mathrm{H}_{5}$ & $\mathrm{H}$ \\
$\mathbf{4 b}$ & $\mathrm{H}$ & $\mathrm{OCH}_{3}$ & $\mathbf{1 0 b}$ & $\mathrm{H}$ & $\mathrm{OCSNHC}_{6} \mathrm{H}_{5}$ \\
$\mathbf{5 a}$ & $\mathrm{OCOCH}_{3}$ & $\mathrm{H}$ & $\mathbf{1 1 a}$ & $\mathrm{NHC}_{3} \mathrm{H}_{7}-\mathrm{n}$ & $\mathrm{H}$ \\
$\mathbf{5 b}$ & $\mathrm{H}$ & $\mathrm{OCOCH}_{3}$ & $\mathbf{1 1 b}$ & $\mathrm{H}$ & $\mathrm{NHC}_{3} \mathrm{H}_{\mathbf{7}}-\mathrm{n}$ \\
$\mathbf{6 a}$ & $\mathrm{OC}_{6} \mathrm{H}_{5}$ & $\mathrm{H}$ & & & \\
$\mathbf{6 b}$ & $\mathrm{H}$ & $\mathrm{OC}_{6} \mathrm{H}_{5}$ & & &
\end{tabular}

MIKE-Spektren ${ }^{7}$ entstehen durch die Zerfallsprodukte von Ionen ausgewähiter Masse mit so geringen Anregungsenergien, daß der Zerfall erst während des Fluges durch den Analysatorteil des doppelfokussierenden Massenspektrometers im 1. feldfreien Raum (nach ca. $5 \times 10^{-6} \mathrm{~s}$ ) oder im 2. feldfreien Raum (nach ca. $10^{-5}$ s) erfolgt. Die Intensitäten der Signale in den MIKESpektren hängen nicht nur von der Struktur der untersuchten Ionen ab, sondern auch von der Verteilungsfunktion der Anregungsenergien, die sie nach ihrer Bildung in der Ionenquelle besitzen ${ }^{8}$. Gleiche MIKE-Spektren für eine Ionensorte aus den Massenspektren verschiedener Verbindungen beweisen daher jeweils gleiche Strukturen für diese Ionen, ungleiche MIKE-Spektren können aber nicht nur durch isobare Ionen verschiedener Struktur, sondern auch durch Ionen gleicher Struktur, aber einer unterschiedlichen Verteilung der Anregungsenergie entstehen. Dieser letzte Fall ist dann zu erwarten, wenn aus zwei Verbindungen gleiche Ionen durch unterschiedliche Reaktionsmechanismen entstehen.

Die MIKE-Spektren der Ionen $m / e=124$ in den 70-eV-Massenspektren von $1 \mathbf{a}, \mathbf{b}-\mathbf{1 1} \mathbf{a}, \mathbf{b}$ wurden für die Zerfälle im 2. feldfreien Raum eines Varian MAT-311A-Massenspektrometers nach der DADI-Technik (DADI $=$ Direct Analysis of Daughter Ions) aufgenom-

${ }^{6}$ F. W. McLafferty, P. F. Bente, R. Kornfeld, S.-C. Tsai und I. Howe, J. Am. Chem. Soc. 95, $2120(1973)$

7) T. W. Shannon und F. W. McLafferty, J. Am. Chem. Soc. 88, 5021 (1966).

8) 8a) A. N. H. Yeo und D. H. Williams, J. Am. Chem. Soc. 93, $395(1971)$. - ${ }^{86)}$ C. W. Tsang und A. G. Harrison, Org. Mass Spectrom. 7, $1377(1973) .{ }^{80)}$ D. J. McAdoo, P. F. Bente III, M. L. Gross und F. W. McLafferty, ebenda 9, 525 (1974). 
men (Tab. 1). Es stimmen nicht nur die MIKE-Spektren der Ionen $m / e=124$ für die einzelnen $\alpha, \beta$-Isomerenpaare innerhalb der Meßgenauigkeit befriedigend überein, sondern auch die für die $\alpha$ - und $\beta$-Isomerenreihe getrennt gemittelten MIKE-Spektren (Tab. 2). Daraus folgt, daß die metastabilen Ionen $m / e=124$ bei $1 \mathbf{a}, \mathbf{b}-\mathbf{1 1} \mathbf{a}, \mathbf{b}$ unabhängig von der Konfiguration an C(3) und der Art des Substituenten gleiche Struktur und weitgehende gleiche Verteilung der Anregungsenergien besitzen. Zur Prüfung, ob die Methode hinreichend empfindlich auf Strukturunterschiede von $\mathrm{C}_{8} \mathrm{H}_{14} \mathrm{~N}^{+}$-Ionen anspricht, wurden die MIKE-Spektren der Ionen $m / e=124$ in den Massenspektren von 5-Allyl-1-methyl2-pyrrolidincarbonitril (12), 2,2,6,6-Tetramethyl-4-piperidinol (13) und 1-Azabicyclo[3.2.2] nonan (14) aufgenommen.<smiles>C=CCC1CCC(C#N)N1C</smiles>

12

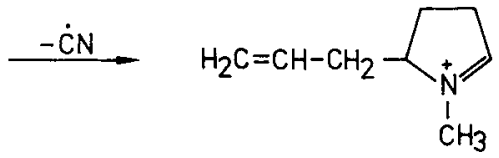

$\mathrm{m} / \mathrm{e} 124$ a<smiles>CC1=CC=CC(C)(C)N1</smiles>

13

$\mathrm{m} / \mathrm{e} 124 \mathrm{c}$

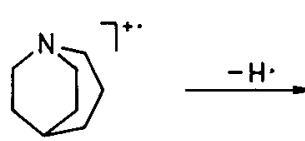

14

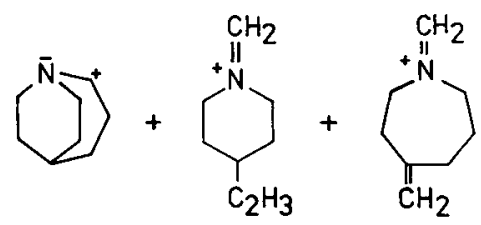

$\mathrm{m} / \mathrm{e} 124 \mathrm{~d}$

e

f

Für die aus den Molekül-Ionen von 12 durch Verlust der CN-Gruppe gebildeten Ionen $m / e=124 \mathrm{kann}$ die Konstitution a angenommen werden. Sie entspricht damit den Ionen, die aus den Molekül-Ionen der Tropanderivate durch Abspaltung des Substituenten nach einer Grob'schen Fragmentierung entstehen. Im Massenspektrum von 13 wird die Konstitution $\mathrm{c}$ für die Ionen $m / e=124$ erwartet, während bei 14 vermutlich ein Ionengemisch $\mathbf{d}-\mathbf{f}$ vorliegt. Die MIKE-Spektren für die Ionen $m / e=124$ von 12-14 sind ebenfalls in Tab. 2 aufgeführt. Man sieht, daß diese Spektren für 13 und 14 deutlich von den übrigen abweichen, wie es für unterschiedliche Ionenstrukturen erwartet wird. Das MIKE-Spektrum der Ionen $m / e=124$ für $\mathbf{1 2}$ stimmt mii denen aus den Tropanderivaten $1-11$ befriedigend überein. Es ist daher anzunehmen, daß auch die Ionen $m / e=124$ bei allen 3-substituierten Tropanen die Konstitution a besitzen, wie sie für eine Grob'sche Fragmentierung der Molekül-Ionen dieser Verbindungen erwartet wird. Da die Ionen jedoch aus den $\alpha$ - und $\beta$-Isomeren mit gleicher Konstitution entstehen, hat die Stereochemie der Molekül-Ionen offenbar keinen Einfluß auf die Konstitution der FragmentIonen. Es kann zunächst nicht ausgeschlossen werden, daß aus den Molekül-Ionen der 


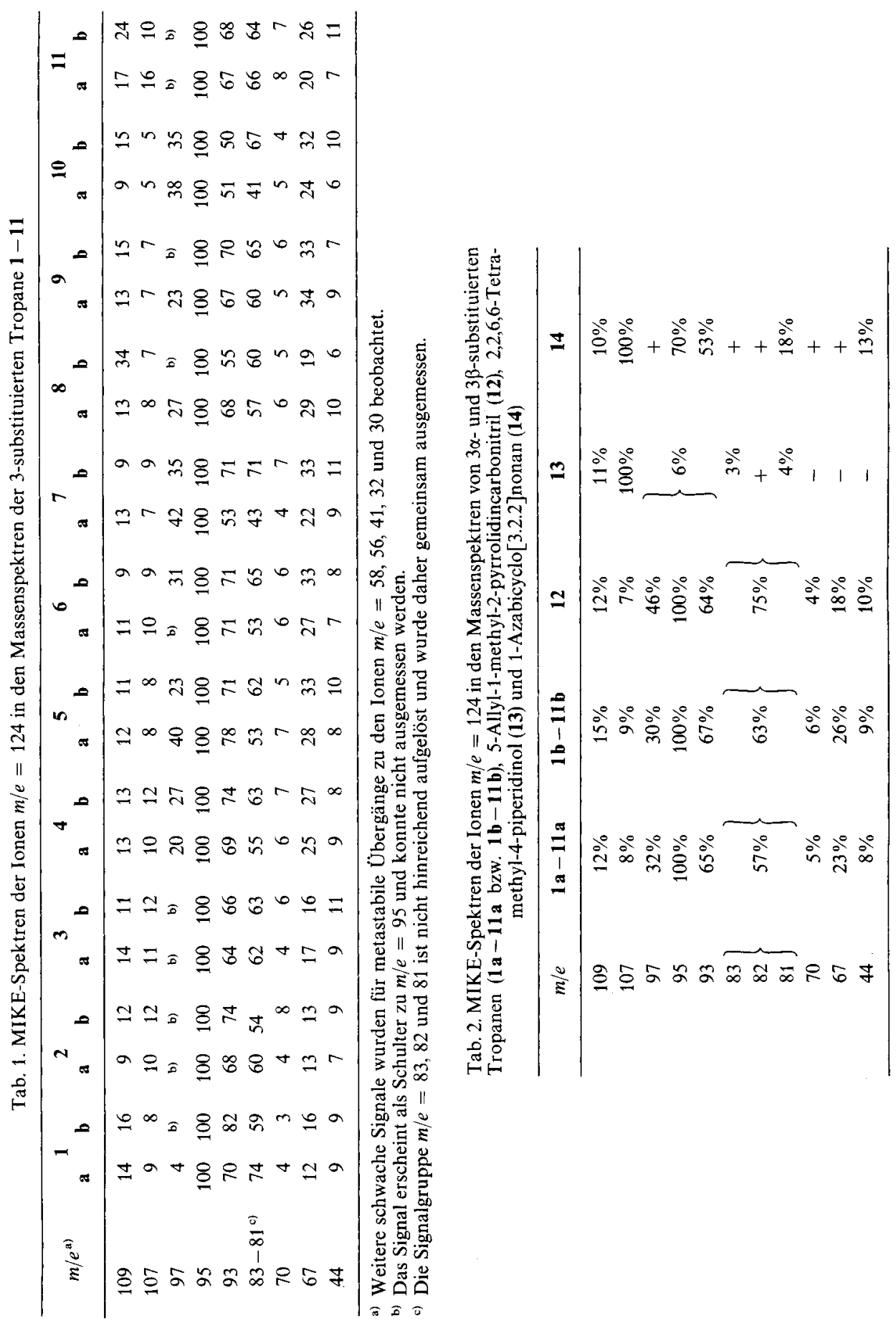




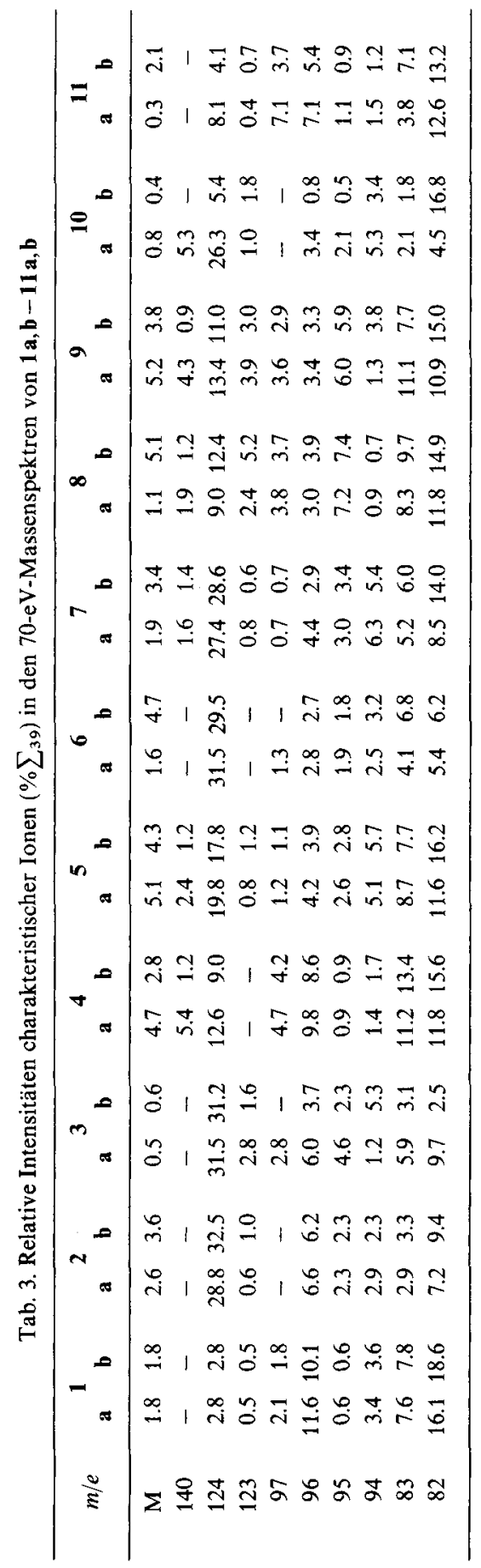


$\alpha$-Isomeren Ionen der Konstitution b (oder ähnlich) entstehen, die sich vor der Weiterreaktion zu a umlagern. Allerdings würde man erwarten, daß diese Ionen $m / e=124$ eine andere Anregungsenergieverteilung besitzen als Ionen a, die direkt aus den MolekülIonen der $\beta$-Isomeren entstehen, so daß aus diesem Grund Unterschiede in den MIKESpektren auftreten sollten ${ }^{8}$. Dies ist aber weder bei den Signalintensitäten der Fall, noch bei den Signalformen, die in allen MIKE-Spektren relativ schmalen Gauß-Formen entsprechen.

Man erwartet, daß bei einer Bildung der Ionen a aus den Molekül-Ionen der $\beta$-Tropanderivate durch einen einstufigen synchronen Prozeß und aus den Molekül-Ionen der $\alpha$ Tropanderivate durch eine zweistufige Reaktion entsprechend dem solvolytischen Verhalten die Intensitäten der Ionen $m / e=124$ in den Massenspektren von $\mathbf{1 b}-\mathbf{1 1} \mathbf{b}$ durchweg deutlich größer sind als bei $1 \mathbf{a}-11$ a. Die $70-\mathrm{eV}$-Massenspektren der $\alpha$ - und $\beta$-Derivate unterscheiden sich deutlich in den Ionenintensitäten. Als Beispiele sind in Abb. 1 und 2 die Massenspektren von $\mathbf{8 a , b}$ und $\mathbf{1 0 a}, \mathbf{b}$ aufgeführt; Tab. 3 enthält die rel. Intensitäten der charakteristischen Ionen in den 70-eV-Massenspektren von 1-11. Quantitative Unterschiede in den Peakmustern der Massenspektren werden für Stereoisomere häufig beobachtet, ohne daß es gelingt, mit hinreichender Sicherheit dafür die Ursachen anzu-
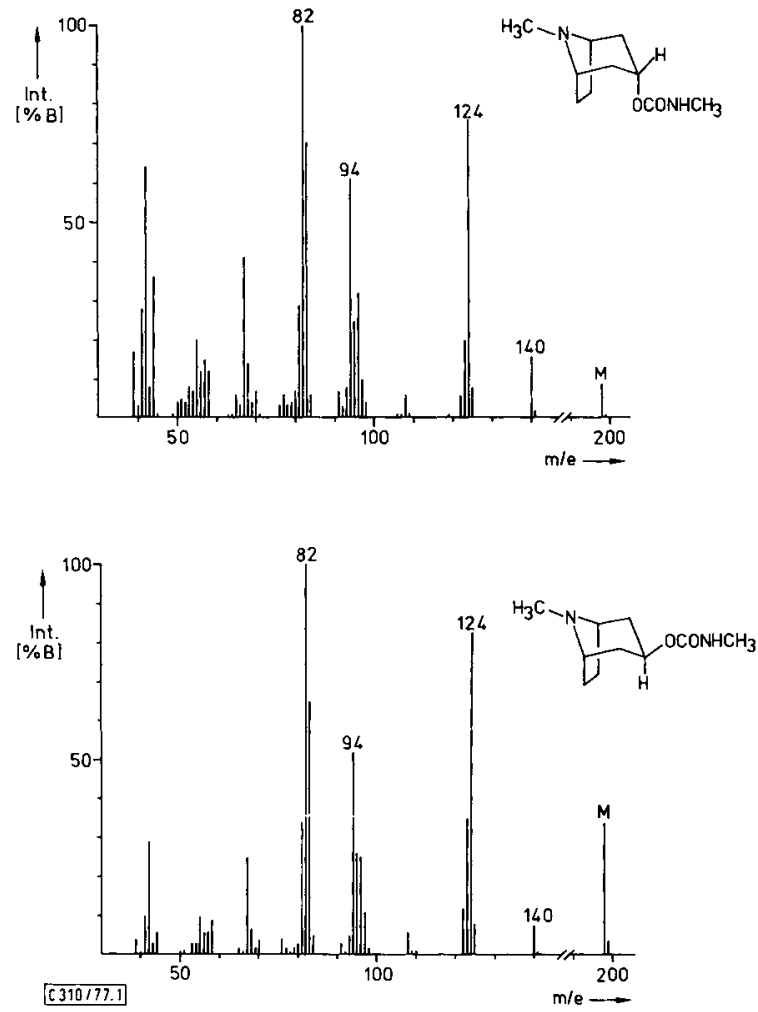

Abb. 1. 70-eV-Massenspektren der Methylcarbamidsäure-3 $\alpha$ - und -3 $\beta$-tropanylester 8a und b $(\% \mathrm{~B}=$ Intensität in $\%$ des Basis-Peaks $)$ 

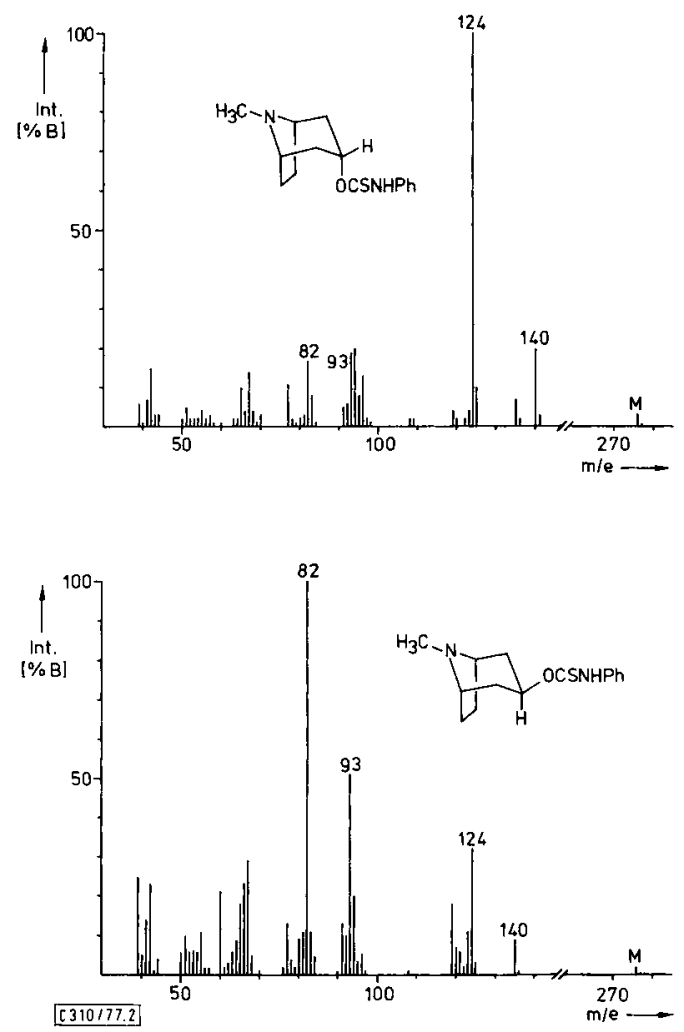

Abb. 2. 70-eV-Massenspektren der (Phenyl)thiocarbamidsäure-3 $\alpha$ - und -3 $\beta$-tropanylester 10a und b $(\% \mathrm{~B}=$ Intensität in $\%$ des Basis-Peaks)

geben ${ }^{9)}$. Bei den 3-substituierten Tropanen beschränken sich die Unterschiede zwischen den Massenspektren der $\alpha$ - und $\beta$-Isomeren keineswegs nur auf die Intensitäten der Ionen $m / e=124$, sondern die Intensitäten der Ionen $m / e=82,83,94-97$ und 140 (bei X $=\mathrm{OR}$ ) variieren in vergleichbarem Ausmaß. Wie an anderer Stelle berichtet wird ${ }^{10)}$, lassen sich diese Unterschiede sowie die MIKE-Spektren der Molekül-Ionen und insbesondere der Ionen $m / e=140$ für eine massenspektrometrische Identifizierung der stereoisomeren Tropanol-Derivate verwenden. Ein Zusammenhang der Intensität der Ionen $m / e=124$ mit der Konfiguration an $\mathrm{C}(3)$ der Tropanderivate wird jedoch nicht gefunden. Je nach Art des Substituenten ist die Intensität dieser Ionen im Massenspektrum des $\alpha$ - oder des $\beta$-Isomeren größer. Es ist daher unwahrscheinlich, daß die Ionen a wie bei solvolytischen Bedingungen aus den $\beta$-Isomeren durch einen schnellen Synchronproze $\beta$ und bei den $\alpha$-Isomeren durch eine zweistufige Reaktion entstehen.

9) M. M. Green, Mass Spectrometry and the Stereochemistry of Organic Molecules in Topics in Stereochemistry, ed. by E. L. Eliel and N. L. Allinger, Vol. 9, Wiley, New York 1976.

10) H.-Fr. Grützmacher und G. Lange, Proceedings of the 4th International Symposium on Mass Spectrometry in Biochemistry and Medicine 1977, im Druck. 
Um weiteren Einblick in die Bildungsreaktion der Ionen $m / e=124$ in der Ionenquelle zu erhalten, wurden die Ionisationspotentiale $I(\mathrm{M})$ und Auftrittspotentiale $A($ Ion) ausgewählter Ionen in den Massenspektren von 1 - 11 gemessen (Tab. 4). Die Werte sind auf ein $I(\mathrm{M})$ des Benzols von $9.5 \mathrm{eV}$ bezogen ${ }^{11)}$. Die $I(\mathrm{M})$-Werte der Tropanderivate liegen mit 7.7-8.3 eV in dem Bereich, der auch für andere bicyclischen Amine gefunden wird ${ }^{12)}$. Die $A(m / e$ 124)-Werte der Ionen $m / e=124$ sind erwartungsgemä $\beta$ von der Natur des abgespaltenen Substituenten $\mathrm{X}$ abhängig. Daneben werden auch Unterschiede zwischen den $A(m / e$ 124) der $\alpha$ - und $\beta$-Isomeren gefunden, die aber kaum außerhalb der Fehlergrenze von $\pm 0.3 \mathrm{eV}$ liegen und zudem keinen systematischen Zusammenhang mit der sterischen Anordnung des Substituenten erkennen lassen. Die Messungen liefern daher gleichfalls keinen Hinweis auf unterschiedliche Bildungsreaktionen der Ionen a aus den Molekül-Ionen der $\alpha$ - und $\beta$-Tropanderivate.

Tab. 4. Ionisationsenergien $I(\mathrm{M})$, Auftrittsenergien $A(m / e$ 124) und scheinbare Bildungsenthalpien der Ionen $m / e=124$ für 3-substituierte Tropane

\begin{tabular}{|c|c|c|c|}
\hline & $\begin{array}{l}I(\mathrm{M})^{\mathrm{a}} \\
(\mathrm{eV})\end{array}$ & $\begin{array}{c}\left.A(m / e 124)^{\mathrm{b}}\right) \\
(\mathrm{eV})\end{array}$ & $\begin{array}{c}\Delta H_{\mathrm{B}}^{\prime}(\mathrm{a}) \\
(\mathrm{kcal} / \mathrm{mol})\end{array}$ \\
\hline $1 \mathbf{a}$ & 8.1 & 10.2 & 177.5 \\
\hline $1 \mathrm{~b}$ & 7.9 & 10.7 & 189 \\
\hline $2 \mathbf{a}$ & 8.1 & 9.1 & 164 \\
\hline $2 b$ & 8.3 & 9.5 & 173.5 \\
\hline $3 \mathbf{a}$ & - & 9.1 & 176 \\
\hline $\mathbf{3 b}$ & 7.8 & 8.9 & 171 \\
\hline $4 a$ & 7.8 & 9.8 & 178 \\
\hline $4 b$ & 7.9 & 10.2 & 186 \\
\hline $\begin{array}{l}5 \mathbf{a} \\
5 \mathbf{b}\end{array}$ & $\begin{array}{l}8.0 \\
8.1\end{array}$ & $\begin{array}{l}10.2 \\
10.3\end{array}$ & $\begin{array}{l}184.5 \\
186\end{array}$ \\
\hline $6 a$ & 8.2 & 8.8 & 174 \\
\hline $\mathbf{6 b}$ & 8.1 & 9.1 & 181 \\
\hline $7 a$ & 7.7 & 9.6 & - \\
\hline $7 \mathrm{~b}$ & - & 9.1 & - \\
\hline $8 \mathbf{a}$ & 7.8 & 10.2 & - \\
\hline $\mathbf{8 b}$ & 8.2 & 9.8 & - \\
\hline $9 a$ & 8.0 & 9.2 & - \\
\hline $10 \mathrm{a}$ & $8^{\text {b) }}$ & 8.6 & - \\
\hline $10 \mathrm{~b}$ & $8^{\text {b) }}$ & 8.4 & - \\
\hline $11 \mathrm{a}$ & 8.1 & 10.5 & - \\
\hline $11 \mathrm{~b}$ & 8.0 & 10.1 & - \\
\hline
\end{tabular}

a) Reproduzierbarkeit $\pm 0.15 \mathrm{eV}$.

b) Reproduzierbarkeit $\pm 0.3 \mathrm{eV}$.

11) J. L. Franklin, J. D. Dillard, H. M. Rosenstock, J. T. Herron und K. Draxl, Ionization Potentials, Appearance Potentials and Heat of Formations of Gaseous Positive Ions, U. S. Dept. of Commerce, Nat. Bureau of Standards, 1969.

12) G. Bieri und E. Heilbronner, Helv. Chim. Acta 57, 546 (1974). 
Aus $A\left(m / e\right.$ 124) läßt sich die Bildungsenthalpie $\Delta H_{\mathrm{B}}$ der Ionen nach (1) berechnen

$$
\begin{aligned}
& A\left(m / e \text { 124) }=\Delta H_{\mathrm{B}}(m / e 124)+E+\Delta H_{\mathrm{B}}\left(\mathrm{X}^{*}\right)-\Delta H_{\mathrm{B}}\left(\mathrm{M}_{\mathrm{X}}\right)\right. \\
& \Delta H_{\mathrm{B}}\left(\mathrm{X}^{*}\right)=\text { Bildungsenthalpie des Radikals } \mathrm{X}^{\cdot} \\
& \Delta H_{\mathrm{B}}\left(\mathrm{M}_{\mathrm{X}}\right)=\text { Bildungsenthalpie des mit } \mathrm{X} \text { substituierten Tropans } \\
& E \quad=\text { Überschußenergie }
\end{aligned}
$$

Da $E$, zu der die kinetische und kompetitive Verschiebung von $A($ Ion), die statistisch verteilte Überschußenergie des aktivierten Komplexes $\varepsilon^{*}$ und die Aktivierungsenergie der Rückreaktion $\varepsilon^{\mathbf{R}}$ Beiträge liefern, nicht bestimmt werden kann, erhält man zunächst nur die scheinbare Bildungsenthalpie $\Delta H_{\mathrm{B}}^{\prime}\left(m / e\right.$ 124) $=\Delta H_{\mathrm{B}}(m / e$ 124) $+E$. Für $1-6$ ist $\Delta H_{\mathrm{B}}\left(\mathrm{X}^{*}\right)$ bekannt ${ }^{13)}, \Delta H_{\mathrm{B}}\left(\mathrm{M}_{\mathrm{X}}\right)$ läßt sich aus Inkrementen berechnen ${ }^{14)}$. Dabei wurde angenommen, daß der Spannungsanteil des Tropanmoleküls mit $12 \mathrm{kcal} / \mathrm{mol} \mathrm{dem} \mathrm{eines}$ Bicyclo[3.2.1] octans entspricht ${ }^{15}$. Außerdem wurden sterische Wechselwirkungen des Substituenten $X$ mit dem Ringgerüst vernachlässigt, so daß für $\alpha$ - und $\beta$-Isomere die gleichen $\Delta H_{\mathrm{B}}\left(\mathrm{M}_{\mathrm{x}}\right)$ erhalten werden. Die auf diese Weise bestimmten $\Delta H_{\mathrm{B}}^{\prime}(m / e 124)$ sind in Tab. 4 mitaufgeführt. Die Werte schwanken vor allem wegen unterschiedlicher Beiträge von $E$ erheblich.

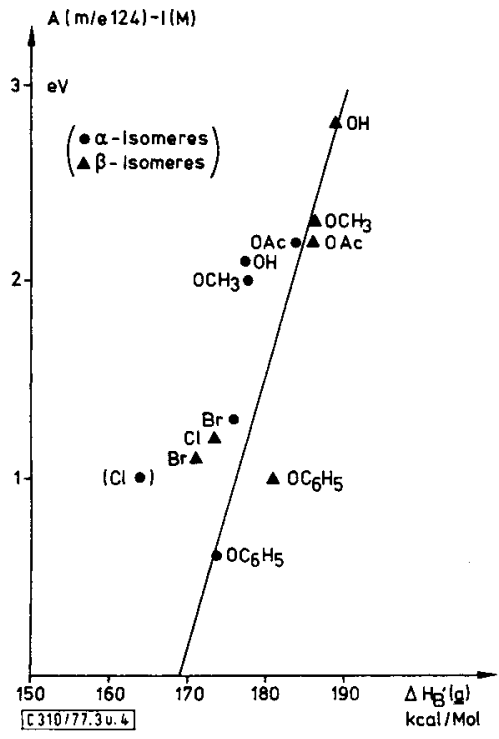

Abb. 3. Beziehung zwischen scheinbarer Aktivierungsenergie $A(m / e$ 124) - I(M) und scheinbarer Bildungsenthalpie $\Delta H_{\mathrm{B}}^{\prime}$ der Ionen $m / e=124$

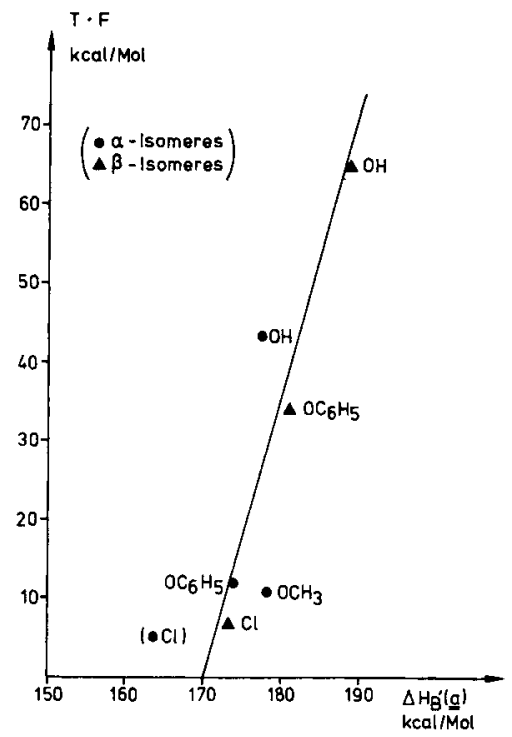

Abb. 4. Beziehung zwischen kinetischer Überschußenergie $T$ und scheinbarer Bildungsenthalpie $\Delta H_{\mathrm{B}}^{\prime}$ der Ionen $m / e=124$

13) K.W. Egger und A. T. Cocks, Helv. Chim. Acta 56, 1516 (1973).

14) S. W. Benson, F. R. Cruickshank, D. M. Golden, G. R. Haugen und H. E. O'Neal, Chem. Rev. 69, 279 (1969).

15) E. M. Engler, J. D. Adose und P. von R. Schleyer, J. Am. Chem. Soc. 95, 8005 (1973). 
Nach Beynon und Mitarbb. ${ }^{16)}$ läßt sich eine Korrektur für $E$ durch zwei empirische Methoden vornehmen unter der Voraussetzung, daß $\varepsilon^{\mathbf{R}}$ klein ist. In diesem Fall wird angenommen, daß $E$ der Differenz $A($ Ion) $-I(\mathrm{M})$ sowie dem Produkt $T \cdot F$ proportional ist (mit $T=$ bei der Fragmentierung freigesetzte kinetische Energie und $F=$ Anzahl der Freiheitsgrade.) Die metastabilen Übergänge für den Zerfall der Molekül-Ionen von 1-6 liefern schmale Signale mit einer Gaußform, so daß $\varepsilon^{\mathrm{R}}$ klein sein muß. Die Abhängigkeit von $H_{\mathrm{B}}^{\prime}(\mathrm{m} / \mathrm{e} 124)$ von $A(\mathrm{Ion})-I(\mathrm{M})$ und $T \cdot F$ ist in Abb. 3 und 4 wiedergegeben. Mit Ausnahme des Wertes für 2a wird eine befriedigende lineare Beziehung gefunden.

Die Extrapolation liefert $\Delta H_{\mathrm{B}}(m / e$ 124) $=169 \mathrm{bzw} .170 \mathrm{kcal} / \mathrm{mol}$ für das $\alpha$ - bzw. $\beta$ Isomere.

$\Delta H_{\mathrm{B}}(\mathrm{a})$ der Ionen a wird mit Hilfe von Inkrement-Werten ${ }^{14)} \mathrm{zu} 176 \pm 3 \mathrm{kcal} / \mathrm{mol}$ berechnet, für die Ionen b wird auf die gleiche Weise $\Delta H_{\mathbf{B}}(\mathbf{b})=189 \pm 4 \mathrm{kcal} / \mathrm{mol} \mathrm{ermittelt}^{17}$. Im Hinblick auf die beträchtlichen Unsicherheiten bei der Bestimmung von $\Delta H_{\mathrm{B}}$-Werten aus massenspektrometrischen Daten und auf die empirischen Korrekturen für $E$ ist die Übereinstimmung des experimentellen Wertes $\Delta H_{\mathrm{B}}(m / e$ 124) von $170 \mathrm{kcal} / \mathrm{mol} \mathrm{mit} \mathrm{dem}$ für die Ionen a berechneten Wert gut. Dies ist ein weiterer Hinweis, daß bei der Fragmentierung der Molekül-Ionen von $\alpha$ - und $\beta$-Tropanderivaten die Ionen a direkt in der Ionenquelle entstehen.

Die Messungen zeigen, daß die Bildung von a nicht die energetisch günstigste Zerfallsreaktion der Molekül-Ionen von $\mathbf{1} \mathbf{a}, \mathbf{b}-\mathbf{1 1} \mathbf{a}, \mathbf{b}$ ist, sondern in der Regel die Bildung der Ionen $m / e=83$ und 82. Diese Ionen entstehen nach einer durch das N-Atom induzierten $\alpha$-Spaltung des Piperidinringes der Molekül-Ionen ${ }^{5}$. Es liegt daher nahe zu vermuten, daß die Aufspaltung zu $\mathrm{M}_{1}^{+\bullet}$ (oder $\mathrm{M}_{2}^{+*}$ ) energetisch sehr günstig ist und schnell erfolgt.
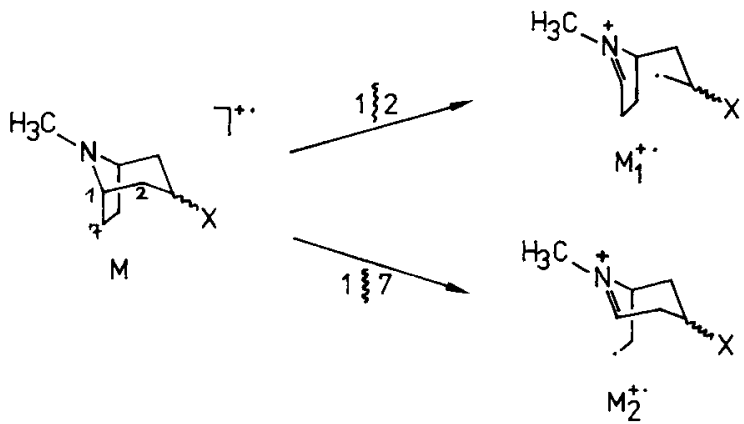

16) J. F. Eder jr., J. H. Beynon u nd R. G. Cooks, Org. Mass Spectrom. 11, 415 (1976).

17) Für die Berechnung von $\Delta H_{\mathrm{B}}(\mathbf{a})$ ist ein Inkrement für die Struktureinheit $\backslash \mathrm{C}=\stackrel{\oplus}{\mathrm{N}}, \mathrm{CH}_{3}$ erforderlich. Es wurde mit Hilfe von $A(\mathrm{M}-\mathrm{H})^{+}=9.5+0.1 \mathrm{eV} \operatorname{der}(\mathrm{M}-\mathrm{H})^{+}$-Ionen des $N$-Methylpyrrolidins auf dem üblichen Wege zu $181 \pm 3 \mathrm{kcal} \mathrm{mol}^{-1}$ bestimmt und enthält zugleich auch den Spannungsanteil für den Fünfring. Für die gleiche Struktureinheit im Sechsring wurde mit Hilfe von $A(\mathrm{M}-\mathrm{H})^{+}$der $(\mathrm{M}-\mathrm{H})^{+}$-Ionen des $N$-Methylpiperidins $186 \pm 3 \mathrm{kcal} \mathrm{mol}^{-1}$ erhalten. Es ist bekannt, daß bei beiden $N$-methylierten cyclischen Aminen das $\mathrm{H}$-Atom aus der $\alpha-\mathrm{CH}_{2}-$ Gruppe der Ringe abgespalten wird ${ }^{18)}$. Zur Berechnung von $\Delta H_{\mathrm{B}}(\mathbf{b})$ wurde das Inkrement für das Strukturelement $\geqslant \mathrm{C}^{\notin}($ Bicyclo[3.2.1]octan) aus $A(\mathrm{M}-\mathrm{Cl})=10.2 \pm 0.15 \mathrm{eV}$ zu $205 \pm 4 \mathrm{kcal} \mathrm{mol}^{-1}$ ermittelt.

18) A. M. Duffield, H. Budzikiewicz, D. H. Williams und C. Dierassi, J. Am. Chem. Soc. 87, 810 (1965). 
Aus den über Inkrementen ${ }^{14,17)}$ zugänglichen $\Delta H_{\mathrm{B}}\left(\mathrm{M}_{1}^{+*}\right)$ und $\Delta H_{\mathrm{B}}\left(\mathrm{M}_{2}^{+\cdot}\right)$ lassen sich die , $A\left(\mathrm{M}_{1}\right)^{\text {"* }}$ und , $A\left(\mathrm{M}_{2}\right)^{\text {t" }}$ zu 8.6 und $8.85 \mathrm{eV}$ abschätzen. Ein Vergleich mit den $A(m / e$ 124) in Tab. 4 zeigt, daß allenfalls bei $10 \mathrm{a}$ und $10 \mathrm{~b}$ die Bildung der Ionen a energetisch ähnlich günstig ist. Da bei der Bildung der modifizierten Molekül-Ionen $\mathbf{M}_{1}^{+\cdot}$ und $\mathbf{M}_{2}^{+*}$ keine Massenänderung erfolgt, kann ihre Entstehung allenfalls über eine Feinstruktur in den Ionenausbeute-Kurven der Molekül-Ionen $\mathrm{M}^{{ }^{+}}$• nachgewiesen werden. Die Bildung von $\mathbf{M}_{1}^{+}$und $\mathbf{M}_{2}^{+\cdot}$ stellt jeweils einen zusätzlichen Prozeß für die Bildung von Ionen mit der Masse der Molekül-Ionen $\mathbf{M}^{+\cdot}$ dar, so daß die IonenausbeuteKurven an den Stellen von $A\left(\mathrm{M}_{1}\right)$ und $A\left(\mathrm{M}_{2}\right)$ Knickpunkte besitzen. Diese Feinstruktur ist bei Aufnahme der Ionenausbeute-Kurven mit den üblichen massenspektrometrischen Ionenquellen wegen der Energieinhomogenität der Stoßelektronen nicht zu erkennen. Collins und Winters ${ }^{19}$ haben mit der EDD-Methode ein mathematisches Verfahren beschrieben, mit dem die Energieinhomogenität ausgeglichen und gegebenenfalls eine Feinstruktur der Ionenausbeute-Kurve erkannt werden kann. Im vorliegenden Fall wurde die ursprüngliche einfache Gleichung (2) benutzt, um die Ionenausbeute-Kurven auf eine Feinstruktur zu untersuchen.

$$
i(\mathrm{~V})=I(\mathrm{~V})-b I(\mathrm{~V}+\mathrm{AV})
$$

In (2) bedeutet $i(\mathrm{~V})$ der um $b I(\mathrm{~V}+\mathrm{AV})$ korrigierte ursprüngliche Ionenstrom $I(\mathrm{~V})$ bei der Elektronenenergie $\mathrm{V} . \Delta \mathrm{V}$ betrug $0.1 \mathrm{eV}, b$ ist ein empirischer Parameter, der von den Ionenquellenbedingungen während der Messung abhängt und für den bei den vorliegenden Messungen durch Austesten Werte zwischen 0.6 und 0.7 ermittelt wurden.

Von den untersuchten Isomerenpaaren liefert nur $\mathbf{5 a}, \mathbf{b}$ hinreichend hohe Intensitäten der Molekül-Ionen, so daß eine Behandlung der Ionenausbeute-Kurven nach dem EDD-Verfahren aussichtsreich erschien ${ }^{20}$. Die EDD-Kurven sind in Abb. 5 zusammen mit der entsprechenden
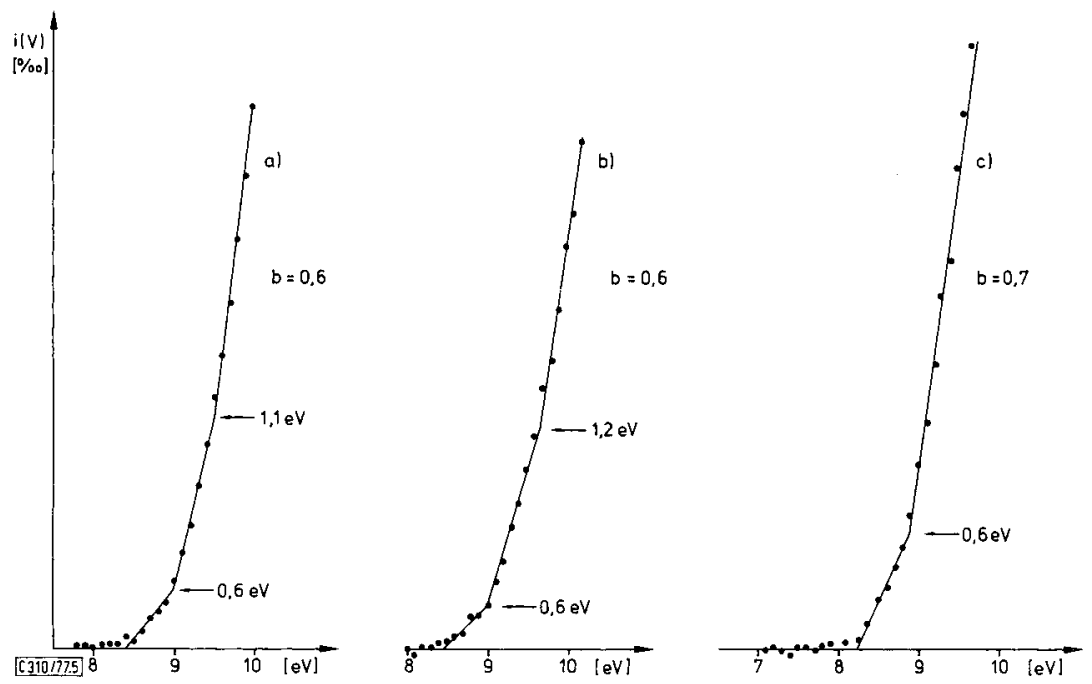

Abb. 5. Ionenausbeutekurven nach der EDD-Methode für die Molekül-Ionen von a) $3 \alpha$-Acetoxy-

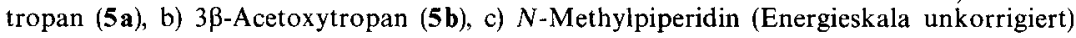

19) E. R. Winters, J. H. Collins und W. L. Courchenne, J. Chem. Phys. 45, 1931 (1966).

20) Als einzelne Isomere wurden auch für $1 \mathbf{a}$ und $\mathbf{4 a}$ die Ionenausbeute-Kurven nach dem EDDVerfahren ausgewertet. Bei 1 a wurden Knickpunkte 0.50 und $1.0 \mathrm{eV}$ oberhaib von $I(\mathrm{M})$ beobachtet, bei $4 \mathrm{a}$ erscheinen Knickpunkte 0.55 und $1.1 \mathrm{eV}$ oberhalb von $I(\mathrm{M})$. 
Kurve für die Moleküi-Ionen von $N$-Methylpiperidin wiedergegeben. In allen drei Fällen erkennt man ca. $0.6 \mathrm{eV}$ oberhalb von $I(\mathrm{M})$ einen deutlichen Knickpunkt, der bei $5 \mathbf{a}, \mathbf{b}$ der hier einsetzenden Bildung von $\mathbf{M}_{1}^{+\cdot}$ und beim $N$-Methylpiperidin der entsprechenden Bildung offenkettiger MolekülIonen durch $\alpha$-Spaltung zugeordnet werden kann. Für $\mathbf{5 a}, \mathbf{b}$ sind jeweils ein weiterer Knickpunkt 1.1 bzw. $1.2 \mathrm{eV}$ oberhalb von $I(\mathrm{M})$ angedeutet. Es ist möglich, daß sich damit die $\alpha$-Spaltung der Molekül-Ionen im Fünfring der Tropanderivate zu erkennen gibt, obwohl die thermochemischen Abschätzungen ein etwas tieferes $A\left(\mathrm{M}_{2}\right)$ für diesen Prozeß erwarten lassen (ca. $0.8-0.9 \mathrm{eV}$ oberhalb der $I(\mathrm{M}))$.

Die Ergebnisse der verschiedenen Untersuchungsmethoden zur Konstitution und Bildung der Ionen $m / e=124$ in den Massenspektren der 3-substituierten Tropane lassen sich widerspruchsfrei zu folgender mechanistischer Beschreibung der Fragmentierung dieser Molekül-Ionen zusammenfassen.

MIKE-Spektren und $\Delta H_{\mathrm{B}}$-Werte der Ionen $m / e=124$ in den Massenspektren der $\alpha$ und $\beta$-Tropanderivate zeigen, daß unabhängig von der Konfiguration an $C(3)$ aus beiden Isomeren Ionen gleicher Konstitution a entstehen. Aus beiden isomeren Molekül-Ionen entstehen die Ionen a durch eine zweistufige Reaktion, die mit der Spaltung der $\mathrm{C}(1)-\mathrm{C}(2)$ Bindung beginnt.
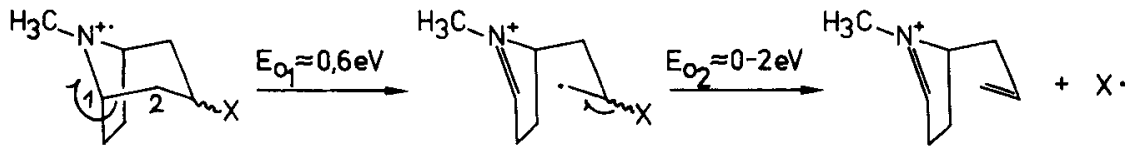

Im zweiten Reaktionsschritt zerfällt das Zwischenprodukt $\mathbf{M}_{1}^{+\bullet}$ weiter durch Abspaltung des Substituenten als Radikal X, wobei dieser Schritt durch die zu X benachbarte Radikalstelle an $\mathrm{C}(2)$ begünstigt wird, je nach der Stabilität von $X^{*}$ jedoch noch einer mehr oder weniger großen Aktivierungsenergie bedarf. Dieser Schritt und damit letztlich die Bildung der Ionen a ist unabhängig von der Konfiguration an $\mathrm{C}(3)$. Hinweise für eine synchrone, durch stereoelektronische Effekte gesteuerte Bildung der Ionen a aus den MolekülIonen werden nicht erhalten. Die quantitativen Unterschiede in den Peakmustern der Massenspektren der $3 \alpha$ - und $3 \beta$-substituierten Tropane werden offenbar durch andere, bisher nur schwer zu deutende sterische Einflüsse hervorgerufen.

Zwischen der solvolytischen und massenspektrometrischen Fragmentierung der hier untersuchten Tropanderivate zu den Ionen a besteht offensichtlich ein fundamentaler Unterschied. Bei der Solvolyse verläßt der Substituent unter Mitwirkung des Lösungsmittels nucleofug das Molekül. Es entsteht ein energiereiches Carbenium-Ion an C(3), dessen energetisch aufwendige Bildung bei geeigneter Orientierung der Bindungsorbitale durch Bildung des Imonium-Ions a vermieden werden kann. Die Aktivierungsenergie für synchrone und zweistufige solvolytische Fragmentierung sind daher unterschiedlich. In den Molekül-Ionen der Tropanderivate kann ein energetisch günstiges Imonium-Ion $\mathbf{M}_{1}^{+\bullet}$ jedoch bereits ohne Spaltung der $\mathrm{C}(3)-\mathrm{X}$-Bindung gebildet werden. Die Aktivierungsenergie hierfür ist für beide Isomere gleich und kleiner als für die Bildung der Ionen a unter zusätzlicher Abspaltung eines Radikals $X$. Sie wird allenfalls durch räumlich sterische Effekte zwischen $X$ und den übrigen Teilen des Moleküls beeinflußt, nicht aber durch stereoelektronische Effekte zwischen den Bindungsorbitalen. 
Diese Arbeit wurde aus Projektmitteln OZ 2142 der Universität Bielefeld unterstützt. Dem Fonds der Chemischen Industrie danken wir für die Unterstützung mit Sachmitteln.

\section{Experimenteller Teil}

Die 70-eV-Massenspektren wurden mit einem Varian MAT 311A-Massenspektrometer bei einer Beschleunigungsspannung von $3 \mathrm{kV}$, einem Elektronenstrom von $2000 \mu \mathrm{A}$ und einer Ionenquellentemperatur von $200^{\circ} \mathrm{C}$ aufgenommen. Feste Substanzen wurden über das Direkteinlaßsystem, flüssige Proben über den auf $150^{\circ} \mathrm{C}$ geheizten Hochtemperatureinlaßteil eingeführt. Bei der Aufnahme der Massenspektren betrug das Auflösungsvermögen $m / \Delta m=1000$, die Bestimmung der Elementzusammensetzung der Ionen erfolgte bei einem Aufösungsvermögen von $m / \Delta m=$ $8000-10000$.

Die MIKE-Spektren wurden am gleichen Gerät und unter den gleichen Bedingungen nach der DADI-Technik aufgenommen.

Die Ionisations- und Auftrittspotentiale wurden mit Hilfe eines Micromass-12B-Massenspektrometers (Firma Vacuum Generators $\mathrm{GmbH}$ ) bei einer Beschleunigungsspannung von $4 \mathrm{kV}$ und einem Elektronenstrom von $20 \mu \mathrm{A}$ ermittelt. Probe und Benzol als Referenzsubstanz wurden gleichzeitig in die Ionenquelle eingelassen und die Ionenausbeute-Kurven in Meßschritten von $0.1 \mathrm{eV}$ mit Hilfe einer automatischen Regelung gemessen. Durch eine magnetische Sprungeinheit können dabei bei konstanter Elektronenenergie bis zu 8 ausgewählte Ionen der Probe und der Referenzsubstanz abgetastet werden, anschließend wird die Elektronenenergie automatisch um $0.1 \mathrm{eV}$ herabgesetzt und der Meßcyclus wiederholt (Cyclusfolgezeit $10 \mathrm{~s}$ ).

${ }^{1} \mathrm{H}$-NMR-Spektren: TMS als innerer Standard, Varian T 60-Gerät mit $\mathrm{CDCl}_{3}$ als Lösungsmittel für die freien Tropane und $\mathrm{D}_{2} \mathrm{O}$ als Lösungsmittel für die Tropan-Hydrochloride.

Dünnschichtchromatographie: Kieselgel (DC-Alu-Folie, Merck, Darmstadt) mit Ethanol/ 25 proz. wäßr. $\mathrm{NH}_{3}(8: 2)$ als Laufmittel. Nachweis mit Dragendorffs Reagenz. Die $R_{\mathrm{F}}-$ Werte sind sehr empfindlich von der $\mathrm{NH}_{3}$-Konzentration abhängig. Deswegen wurden relative $R_{\mathrm{F}}$-Werte bestimmt, bezogen auf $3 \alpha$-Tropanol (1 a) als Referenz.

2,2,6,6-Tetramethyl-4-piperidinol (13), 1-Azabicyclo[3.2.2]nonan (14), $3 \alpha$-Tropanol (1 a) sowie Tropinon wurden aus dem Handel bezogen und ohne weitere Reinigung für die Versuche eingesetzt.

5-Allyl-1-methyl-2-pyrrolidincarbonitril (12) wurde nach Lit. ${ }^{21)}$ aus $3 \beta$-Chlortropan und Natriumcyanid dargestellt. Sdp. $73-75^{\circ} \mathrm{C} / 2$ Torr (Lit. ${ }^{21)} 106-108^{\circ} \mathrm{C}$ ).

$3 \beta$-Tropanol (1 b) wurde durch Reduktion von Tropinon mit Natrium in Isopropylalkohol erhalten ${ }^{22)}$. Ausb. $65 \%$, Schmp. $107^{\circ} \mathrm{C}$ (Lit. ${ }^{22)} 106-108^{\circ} \mathrm{C}$ ).

Die Tropanole $1 \mathbf{a}$ und $1 \mathbf{b}$ dienten als Ausgangssubstanzen für die Darstellung aller 3-substituierten Tropanderivate.

$3 \alpha$-Chlortropan (2a), als Hydrochlorid Schmp. $170-173^{\circ} \mathrm{C} / 220-223^{\circ} \mathrm{C}$ (Lit. ${ }^{4)} 170-171^{\circ} \mathrm{C} /$ $225-227^{\circ} \mathrm{C}$ ).

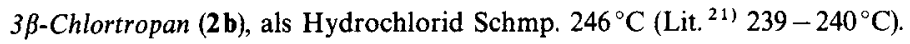

3 $\alpha$-Bromtropan (3a), als Hydrobromid Schmp. $140-150^{\circ} \mathrm{C}$ (unscharf, Lit. ${ }^{23)} \mathrm{ca} .150^{\circ} \mathrm{C}$ ).

$3 \beta$-Bromtropan (3b), als Hydrobromid Schmp. $210-218^{\circ} \mathrm{C}$ (Lit. ${ }^{23)} 215-220^{\circ} \mathrm{C}$ ).

$3 \alpha$-Methoxytropan (4a), Sdp. $97^{\circ} \mathrm{C} / 24$ Torr (Lit. ${ }^{24)} 82^{\circ} \mathrm{C} / 15$ Torr).

21) S. Archer, T. R. Lewis und B. Zenitz, J. Am. Chem. Soc. 80, 958 (1958).

22) A. Nickon und L. F. Fieser, J. Am. Chem. Soc. 74, 5566 (1952).

${ }^{23)} P$. Schreiber, G. Kraiss und K. Nador, J. Chem. Soc. B 1970, 1366.

24) G. Kraiss, P. Schreiber und K. Nador, J. Chem. Soc. B 1971, 2145. 
3ß-Methoxytropan (4b), Sdp. $92^{\circ} \mathrm{C} / 12$ Torr (Lit. ${ }^{25)} 90^{\circ} \mathrm{C} / 15$ Torr).

$3 \alpha$-Acetoxytropan (5a), Sdp. $112-114^{\circ} \mathrm{C} / 17$ Torr (Lit. ${ }^{26)} 125-128^{\circ} \mathrm{C} / 25$ Torr).

$3 \beta$-Acetoxytropan (5b), Sdp. $117-118^{\circ} \mathrm{C} / 19$ Torr (Lit. ${ }^{26)} 118-120^{\circ} \mathrm{C} / 17$ Torr)

$3 \alpha$-Phenoxytropan (6a), Schmp. $50^{\circ} \mathrm{C}$ (Lit. ${ }^{25)} 53^{\circ} \mathrm{C}$ ).

33-Phenoxytropan (6b), Schmp. $48^{\circ} \mathrm{C}$ (Lit. ${ }^{25)} 51^{\circ} \mathrm{C}$ ).

$3 \alpha-($ Methylsulfonyloxy)tropan (7a) wurde durch Umsetzen von 1 a mit Methansulfonylchlorid nach Lit. ${ }^{27)}$ als Hydrochlorid dargestellt. Mehrfaches Umfällen aus absol. Ethanol/Ether lieferte ein chromatographisch reines Produkt, das jedoch hygroskopisch war und keinen definierten Schmp. besaß. Ausb. $49 \%$.

$$
\mathrm{C}_{9} \mathrm{H}_{17} \mathrm{NO}_{3} \mathrm{~S} \quad \text { Ber. } 219.0929 \text { Gef. } 219.0925 \text { (massensepktrometr.) }
$$

$3 \beta-($ Methylsulfonyloxy) tropan (7b) aus $\mathbf{1} \mathbf{b}$ analog zu 7 a als Hydrochlorid. Nach Umkristallisieren aus Ethanol/Ether Ausb. $21 \%$, Schmp. $203-206^{\circ} \mathrm{C}$ (Zers.).

$$
\mathrm{C}_{9} \mathrm{H}_{1}{ }_{7} \mathrm{NO}_{3} \mathrm{~S} \text { Ber. } 219.0929 \text { Gef. } 219.0921 \text { (massenspektrometr.) }
$$

\begin{tabular}{|c|c|c|c|c|}
\hline Verbindung & $\delta$ & $\begin{array}{l}\text { Signalform/ } \\
\text { Signalbreite }(\mathrm{Hz})\end{array}$ & $\begin{array}{l}\text { Kopplungs- } \\
\text { konst. (Hz) }\end{array}$ & rel. $R_{\mathrm{F}}$ \\
\hline $1 \mathbf{a}$ & 4.02 & Pseudotriplett, 18 & 5 & a) \\
\hline lb & 3.92 & Nonett, 38 & 10 und 7 & 1.5 \\
\hline $2 a$ & 4.35 & Pseudotriplett, 18 & 6 & 2.0 \\
\hline $2 \mathbf{b}$ & 4.12 & Nonett, 39 & 10 und 8 & 2.2 \\
\hline 3a & - & - & - & 1.6 \\
\hline $3 \mathbf{b}^{\text {b) }}$ & 4.55 & Nonett, 40 & 10 und 7.5 & 2.9 \\
\hline $4 a$ & 3.39 & Pseudotriplett, 18 & 3.5 & 1.4 \\
\hline $4 \mathrm{~b}$ & - & - & - & 1.7 \\
\hline $5 \mathbf{a}$ & 5.01 & Pseudotriplett, 17 & 5 & 2.7 \\
\hline $5 b$ & 5.14 & Nonett, 39 & 9.5 und 8 & 3.5 \\
\hline $6 a$ & 4.48 & unaufgelöst, 18 & - & 1.7 \\
\hline $6 \mathrm{~b}$ & 4.44 & Nonett, 37 & 9 und 7 & 2.5 \\
\hline $7 a^{b)}$ & 5.08 & unaufgelöst, 18 & - & 1.6 \\
\hline $7 b^{b)}$ & 5.15 & Nonett, 38 & 10 und 6.5 & 1.9 \\
\hline $8 \mathbf{a}$ & 4.90 & Pseudotriplett, 18 & 5 & 1.8 \\
\hline $8 b$ & 4.93 & Nonett, 39 & 9.5 und 7.5 & 2.5 \\
\hline 9a & 5.02 & Pseudotriplett, 18 & 5 & 1.9 \\
\hline $9 \mathrm{~b}$ & 5.05 & Nonett, 39 & 10 und 7 & 2.5 \\
\hline $10 \mathrm{a}$ & 5.60 & Pseudotriplett, 18 & 5 & 1.9 \\
\hline $10 \mathrm{~b}$ & 5.70 & Nonett, 39 & 11 und 6.5 & 2.1 \\
\hline
\end{tabular}

Tab. 5. ${ }^{1} \mathrm{H}-\mathrm{NMR}$-Daten (chem. Verschiebungen $\delta$ ) und relative $R_{\mathrm{F}}$-Werte ${ }^{1)}$ der 3-Tropanderivate

a) Bezogen auf $3 \alpha-$ Tropanol $(1 \mathrm{a}), \mathrm{R}_{\mathrm{g}} \mathbf{1} \mathrm{a} \approx 0.4$.

b) Als Hydrochlorid bzw. Hydrobromid gemessen.

${ }^{25)}$ G. Kraiss, P. Schreiber und K. Nador, J. Org. Chem. 33, 2601 (1968).

26) J. B. Kay, J. B. Robinson und J. Thomas, J. Chem. Soc. B 1965, 5112.

27) S. Archer, M. R. Bell, T. R. Lewis, J. W. Schulenburg und M. J. Unser, J. Am. Chem. Soc. 80, 4677 (1958). 
Die Carbamidsäure-tropanylester $\mathbf{8 a}, \mathbf{b}-\mathbf{1 0} \mathbf{a}, \mathbf{b}$ wurden nach einer Vorschrift von Sekera ${ }^{28}$ ) aus 1 a bzw. 1 b durch Umsetzen mit den entsprechenden Isocyanaten bzw. Phenylisothiocyanat erhalten und mehrfach aus Ethanol umkristallisiert. Die Produkte waren dünnschichtchromatographisch einheitlich.

Methylcarbamidsäure-3 $\alpha$-tropanylester (8a): Schmp. $130-131^{\circ} \mathrm{C}$.

$$
\mathrm{C}_{10} \mathrm{H}_{18} \mathrm{~N}_{2} \mathrm{O}_{2} \text { Ber. 198.1368 Gef. } 198.1368 \text { (massenspektrometr.) }
$$

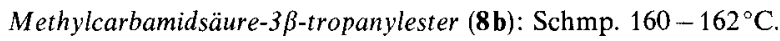

$$
\mathrm{C}_{10} \mathrm{H}_{18} \mathrm{~N}_{2} \mathrm{O}_{2} \text { Ber. } 198.1368 \text { Gef. } 198.1364 \text { (massenspektrometr.) }
$$

Phenylcarbamidsäure-3 $\alpha$-tropanylester (9a): Schmp. $170-173^{\circ} \mathrm{C}$.

$$
\mathrm{C}_{15} \mathrm{H}_{20} \mathrm{~N}_{2} \mathrm{O}_{2} \text { Ber. } 260.1527 \text { Gef. } 260.1494 \text { (massenspektrometr.) }
$$

Phenylcarbamidsäure-3 $\beta$-tropanylester (9b): Schmp. $200-203^{\circ} \mathrm{C}$.

$$
\mathrm{C}_{15} \mathrm{H}_{20} \mathrm{~N}_{2} \mathrm{O}_{2} \text { Ber. } 260.1527 \text { Gef. } 260.1495 \text { (massenspektrometr.) }
$$

(Phenyl)thiocarbamidsäure-O-3 $\alpha$-tropanylester (10a): Schmp. $148-153^{\circ} \mathrm{C}$.

$\mathrm{C}_{15} \mathrm{H}_{20} \mathrm{~N}_{2} \mathrm{OS}$ Ber. 276.1296 Gef. 276.1293 (massenspektrometr.)

(Phenyl)thiocarbamidsäure-O-3ß-tropanylester (10b): Schmp. $183^{\circ} \mathrm{C}$.

$$
\mathrm{C}_{15} \mathrm{H}_{20} \mathrm{~N}_{2} \mathrm{OS} \text { Ber. 276.1296 Gef. } 276.1296 \text { (massenspektrometr.) }
$$

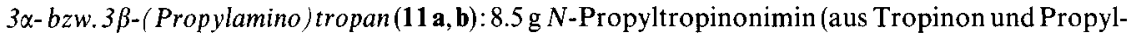
amin) wurden mit $4.5 \mathrm{~g}$ Natrium in Ethanol zum Isomerengemisch $11 \mathbf{a}, \mathbf{b}$ hydriert. Nach Abdampfen des Lösungsmittels i. Vak. lieferte eine Destillation des Rohproduktes $4.6 \mathrm{~g}(54 \%) 11 \mathrm{a}, \mathrm{b}$, Sdp. $100-115^{\circ} \mathrm{C} / 16$ Torr. Die Trennung der Isomeren erfolgte durch Säulenchromatographie (Kieselgel, Laufmittel Aceton/ $\mathrm{CH}_{2} \mathrm{Cl}_{2} / 25$ proz. wäßr. $\left.\mathrm{NH}_{3} 8: 1: 1\right)$ und erneute Destillation i. Vak.

$3 \alpha$-(Propylamino)tropan (11 a): Sdp. $108-114^{\circ} \mathrm{C} / 25$ Torr.

$$
\mathrm{C}_{11} \mathrm{H}_{22} \mathrm{~N}_{2} \text { Ber. } 182.1783 \text { Gef. } 182.1767 \text { (massenspektrometr.) }
$$

$3 \beta$-( Propylamino )tropan (11 b): Sdp. $97-105^{\circ} \mathrm{C} / 15$ Torr.

$$
\mathrm{C}_{11} \mathrm{H}_{22} \mathrm{~N}_{2} \text { Ber. 182.1783 Gef. } 182.1770 \text { (massenspektrometr.). }
$$

28) A. Sekera, Ann. Chim. 7, 537 (1962). 